\title{
Criteria for Glass-Forming Ability Accessible by Molecular Dynamics Simulations
}

\author{
Masato Shimono and Hidehiro Onodera \\ National Institute for Materials Science, Tsukuba 305-0047 Japan
}

Criteria for estimating the glass-forming ability accessible by the molecular dynamics simulations are proposed. The enthalpy of the glassy phases and the density of the supercooled liquid phases can be the criteria, the former reflects the energetics and the latter reflects the dynamics. The relaxation behavior of the glassy phases suggests that the cooling rate dependence of the glass transition temperature also could be the criterion as to explore the 'energy landscape' and the phase stability for both supercooled liquid and glassy phases.

(Received December 5, 2003; Accepted March 12, 2004)

Keywords: metallic glass, supercooled liquid, computer simulation, rapid solidification, diffusion

\section{Introduction}

The discovery ${ }^{1,2)}$ of bulk metallic glasses has widened the application fields of glassy materials because of a wide temperature range of stable supercooled liquid region in the system. However, it is still a hard task to explore new glassy materials experimentally by selecting constituent elements among thousands of combinations. Therefore, it is useful for us with respect to the materials design if we could predict appropriate alloy systems with high glass-forming ability by the use of computer simulations.

Among miscellaneous simulation methods, the molecular dynamics (MD) simulation is a powerful tool for investigating many aspects of phenomena at the atomistic scale. However, the present capacity of computers allows us to handle time scale in the order of nanoseconds at a maximum in the framework. This fact leads us to difficulties in estimating the glass-forming ability of alloy systems by using the MD simulations.

As good indicators for the glass-forming ability, there are three well-known parameters: ${ }^{3)}$ the critical cooling rate $R_{\mathrm{c}}$ needed for glass-formation, the width of supercooled liquid region $\Delta T=T_{\mathrm{x}}-T_{\mathrm{g}}$, and the reduced glass transition temperature $T_{\mathrm{g}} / T_{\mathrm{m}}$. Here $T_{\mathrm{m}}$ is the melting or the liquidus temperature of the stable crystalline phase, $T_{\mathrm{x}}$ is the crystallization temperature of the glassy phase, and $T_{\mathrm{g}}$ is the glass transition temperature. In the MD simulations, all those three parameters $\left(R_{\mathrm{c}}, \Delta T\right.$, and $\left.T_{\mathrm{g}} / T_{\mathrm{m}}\right)$ are difficult to estimate because of the following reasons.

First, the typical cooling rate that can be achieved by MD simulations is $10^{12} \mathrm{~K} / \mathrm{s}$. This value is extremely higher than the critical cooling rate $R_{\mathrm{c}}$ for the bulk metallic glasses, which lies in the order of $\mathrm{K} / \mathrm{s}$ or less. The discrepancy of $10^{12}$ between them is one of the serious problems, and can be understood by a simple model of alloys, which has been investigated in the preceding MD study. ${ }^{4)}$ In Fig. 1, we have shown the glass-forming ranges under rapid solidification calculated by MD simulations for a set of binary systems with different atomic size ratios. The horizontal axis denotes the concentration of alloying elements and the vertical one denotes the atomic size ratio of alloying elements. Figure 1 shows the tendency to decrease the glass-forming range with decreasing cooling rate. The binary systems with sufficiently large atomic size difference show a wide glass-forming range

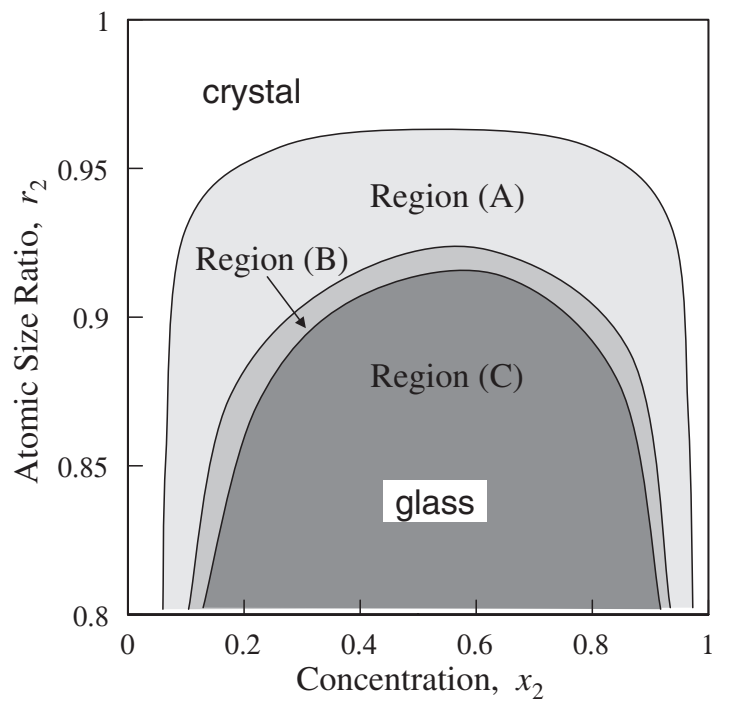

Fig. 1 Calculated glass-forming ranges for the model binary system under rapid cooling at different cooling rates. The regions (A), (B), and (C) correspond to the calculation results at cooling rate of $2 \times 10^{-3}, 2 \times 10^{-4}$, and $2 \times 10^{-5}$, respectively. All units are normalized by the LJ parameters.

even in the lowest cooling rate accessible in the MD simulation. Therefore, we cannot point out the locus with the highest glass-forming ability in the region (C). Consequently, the MD simulation has little predictive power for estimating the glass-forming ability.

Secondly, owing to the fact that the maximum annealing time is in the order of nanoseconds in the MD simulation, we can hardly detect crystallization for any systems with high glass-forming ability exemplified by the region (C) in Fig. 1. Hence $T_{\mathrm{x}}$ at that composition cannot be estimated by the simulation, neither can $\Delta T$.

Finally, in the above circumstances, we cannot identify the thermodynamically stable crystalline phase at the composition. Thus, we neither can estimate $T_{\mathrm{m}}$, which should be defined by the melting temperature of the stable crystalline phase. This implies we cannot estimate $T_{\mathrm{g}} / T_{\mathrm{m}}$ even though we could know $T_{\mathrm{g}}$.

In the present study, we propose some criteria for evaluation of the glass-forming ability that are accessible by MD simulations. In addition, the relaxation behavior of the supercooled liquid and glassy phases, which is expected 
to be related to the criteria, is also examined at the atomistic scale in connection with the energy landscape theory. ${ }^{5,6)} \mathrm{We}$ hope that our suggestions would offer a clue to overcome the barrier in time scale of $10^{12}$ between experiments and simulations.

\section{Computational Methods}

We consider a binary system with 4000 atoms under a periodic boundary condition. To evaluate the glass-forming ability of the system, we simulate rapid solidification processes from the liquid phase. The pressure of the system is kept zero by using the constant-pressure formalisms ${ }^{7)}$ and the temperature of the system is controlled by scaling the atomic momenta.

The interaction between the elements $i$ and $j$ is described by the 8-4 Lennard-Jones (LJ) potential:

$$
V_{i j}(r)=e_{i j}\left\{\left(r_{i j} / r\right)^{8}-2\left(r_{i j} / r\right)^{4}\right\},
$$

which is proved ${ }^{8)}$ to be adequate for metallic systems. The potential expressed by eq. (1) has the minimum $-e_{i j}$ at the distance $r_{i j}$, which can be considered as the chemical bond strength and the atomic size, respectively. The glass-forming ability of this system under rapid solidification has been investigated in the earlier MD study, ${ }^{4}$ and it was reported that the geometrical factor plays a decisive role. Accordingly, we here investigate a binary system composed of elements 1 and 2 with equi-chemical interactions for simplicity. In the present study, the following parameters are dealt with as constant: $r_{11}=1$ and $e_{11}=e_{22}=e_{12}=1$. On the other hand, the atomic size ratio $r_{2} \equiv r_{22} / r_{11}$ and the concentration $x_{2}$ of the element 2 are the variables. The interactive distance $r_{12}$ between different elements is varied as to satisfy the relation $r_{12}=\left(r_{11}+r_{22}\right) / 2$. All physical quantities used in the present study are expressed in the above units throughout this paper.

The melting temperature $T_{\mathrm{m}}$ of the pure system $\left(x_{2}=0\right.$ or 1) was calculated ${ }^{4)}$ to be around 0.67 in the above-mentioned dimensionless units. As starting liquid phases, we prepare binary mixtures at $T=0.7$ with various atomic size ratio $r_{2}$ ranging from 0.8 to 1 and composition $x_{2}$ ranging from 0 to 1 , and then cool down the system to solidify.

\section{Energy Landscape Theory}

An attractive approach for understanding the glass-forming phenomena from a viewpoint of physics, which is called the energy landscape theory, has been proposed by Stillinger. ${ }^{5,6)}$ Recently, concrete evidence for this theory was obtained $^{9)}$ by computer simulations of a model glass-forming system. The energy landscape theory offers a convenient viewpoint for the analyses of supercooled liquids and glassy states. Therefore, we shall illustrate how supercooling and glass transition can be interpreted in the framework of this theory.

Figure 2 is a schematic view of the 'energy landscape' of a system. The horizontal axis denotes the configuration space of all atoms in the system and the vertical axis denotes the internal energy of the whole system. The dynamics of the system can be viewed as a motion in this configuration space.

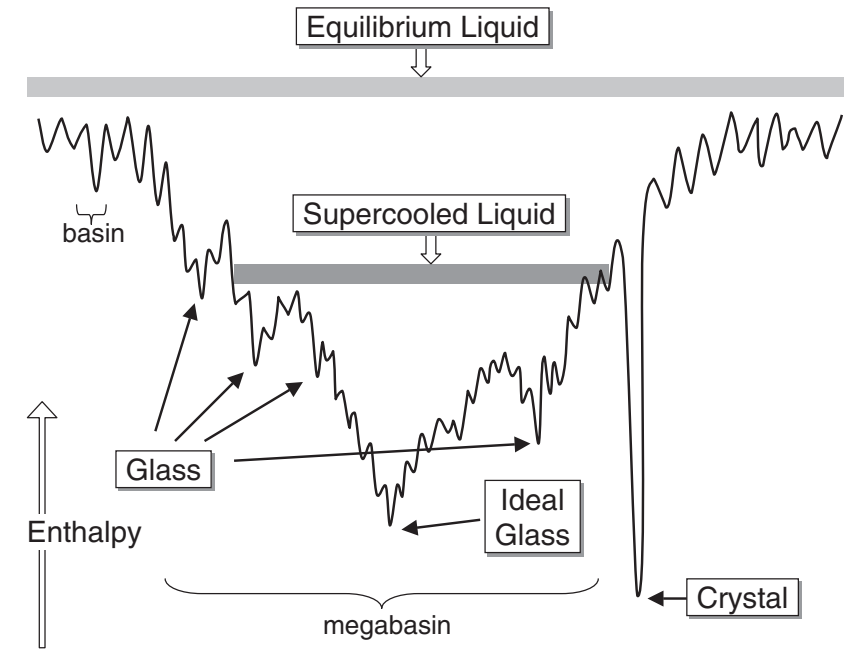

$=$ Atomic Configuration $\Longrightarrow$

Fig. 2 The topographical view of an energy landscape. The horizontal axis denotes the configuration space of all atoms in the system and the vertical axis denotes the total enthalpy of the whole system.

The innumerous potential minima are called 'basins', one of which corresponds to the stable crystalline state and some of which correspond to different states of glassy phase. Among the glassy basins, the basin with the lowest energy might be considered as the 'ideal glass', if it exists.

According to the energy landscape theory, equilibrium liquid phases can cover all over the energy landscape, while a supercooled liquid phase can cover a part of the landscape, which is understood as a trapped state into one bunch of basins or a crater called 'megabasin' as depicted in Fig. 2. A glassy state is understood as a trapped state into one of basins. In Fig. 2, the number of basins means the number of inherent structure of the glassy phase, i.e., the configurational entropy. If we classify the basins by their enthalpy, the number $\Omega(E)$ of basins as a function of the enthalpy $E$ should decrease monotonically as decreasing $E$. The basin with the lowest energy $E_{0}$ aside from those of crystalline phases corresponds to the ideal glassy state, where the configurational entropy vanishes, or $\Omega\left(E_{0}\right)=1$. The simulation results obtained in the present study, which support the energy landscape theory, will be shown in Section 4.2.3.

\section{Results}

\subsection{Simulation Procedures}

We can examine the properties of emerging phases during the simulation by monitoring the observables such as the atomic volumes, the internal energy (enthalpy), the radial distribution function, and the atomic configuration. Figure 3 shows the time evolution of the atomic volume of the $\left(r_{2}, x_{2}\right)$ $=(0.9,0.3)$ system during cooling procedures from the liquid phase at different cooling rates $\left(2 \times 10^{-4}\right.$ and $\left.2 \times 10^{-5}\right)$. Here the closed symbols and the open symbols correspond to the higher and the lower cooling rate, respectively. As seen from the atomic configurations shown in the insets in Fig. 3, we have got a crystalline phase for the slower cooling rate, while a glassy phase for the faster cooling rate. The glass 


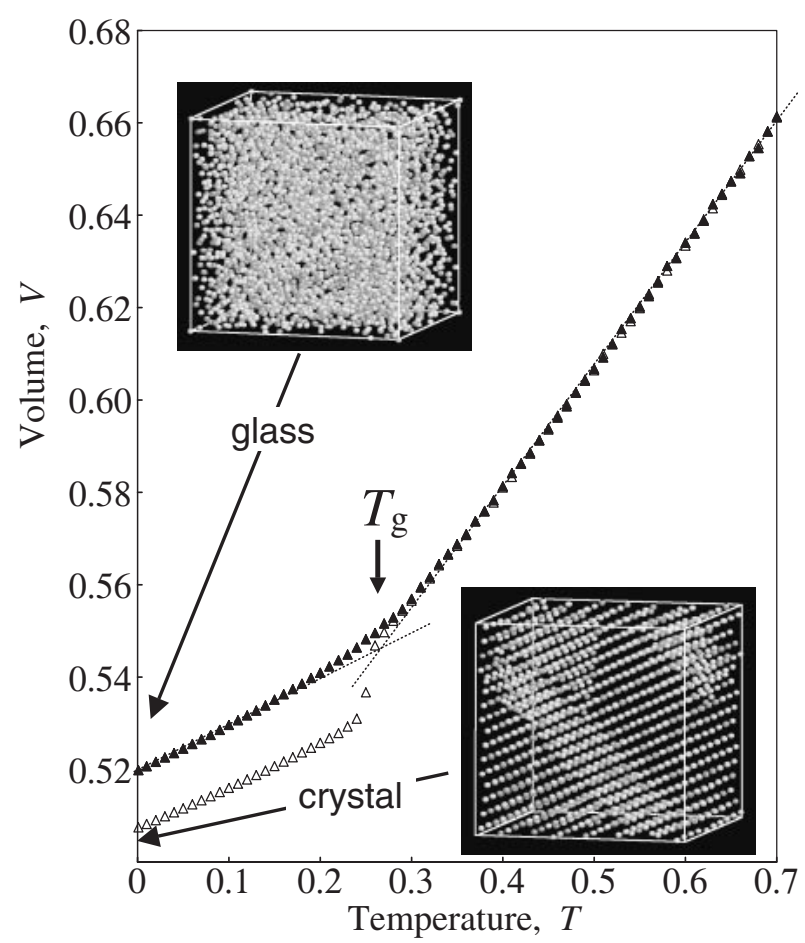

Fig. 3 The time evolution of the atomic volume of the $\left(r_{2}, x_{2}\right)=(0.9,0.3)$ system under rapid solidification at different cooling rates, where the closed triangles and the open triangles correspond to the higher cooling rate $\left(2 \times 10^{-4}\right)$ and the lower rate $\left(2 \times 10^{-5}\right)$, respectively. The insets show the snapshots of the atomic configuration for the solidified phases.

transition temperature $T_{\mathrm{g}}$ is determined by the discontinuity of the slope as marked in Fig. 3. Thus, we can estimate the glass-forming range under rapid solidification at a constant cooling rate as already shown in Fig. 1.

Here we should note the order of the cooling rate achieved in this MD study. Supposing that the element 1 is identified with $\mathrm{Ni}$, that is, the length unit is to be the atomic size $0.25 \mathrm{~nm}$ of $\mathrm{Ni}$ atom, the mass unit is to be the atomic mass 58.7 a.u. of $\mathrm{Ni}$, and the energy unit to be taken as to reproduce the melting temperature $1726 \mathrm{~K}$ of $\mathrm{Ni}$, then the unit of cooling rate becomes about $5 \times 10^{15} \mathrm{~K} / \mathrm{s}$. Therefore, two different cooling rates $2 \times 10^{-4}$ and $2 \times 10^{-5}$ found in Fig. 3 approximately corresponds to $10^{12} \mathrm{~K} / \mathrm{s}$ and $10^{11} \mathrm{~K} / \mathrm{s}$, respectively, in this case.

\subsection{Stability of liquid and glassy phases}

In this section, we shall evaluate the glass-forming ability by estimating the phase stability of liquid and glassy phases in the system because of the difficulties in directly estimating the glass-forming ability, which is mentioned in Section 1.

Phase stability of the supercooled liquid phase or the glassy phase is generally determined by several factors such as the free energy difference between competing phases, the energy barrier on the transition path to crystalline phases, and the rate of atomic diffusion needed for the transformation to the lower energy states. Here, it is important to note that we could hardly have the information of the competing crystalline phases, because we can scarcely observe crystallization phenomena in most of the composition range such as the region $(\mathrm{C})$ in Fig. 1 within the finite simulation time. In order

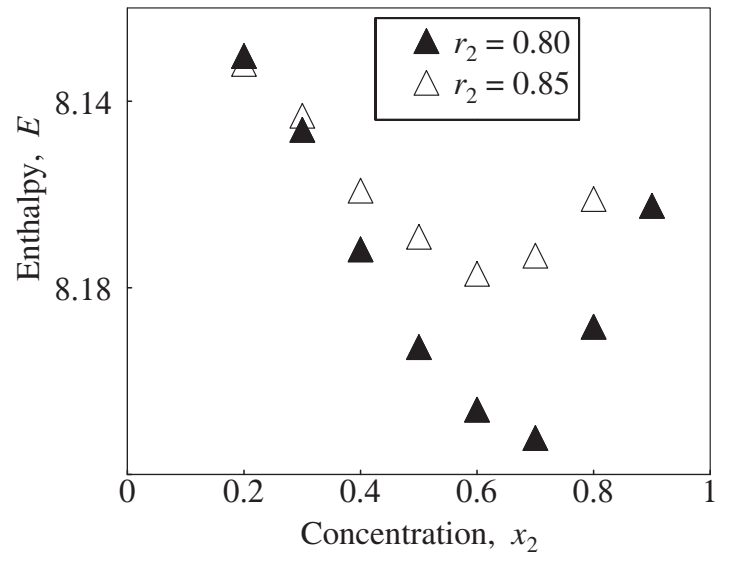

Fig. 4 The concentration dependences of the atomic enthalpy of the asquenched glassy phases for two systems with different atomic size ratios, where the closed symbols and the open symbols correspond to the atomic size ratio 0.8 and 0.85 , respectively.

to overcome the restrictions mentioned above, we shall focus on the following three properties, that is, the enthalpy of glassy phases, the diffusivity of liquid phases, and the relaxation behavior of glassy and supercooled liquid phases.

\subsubsection{Enthalpy of glassy phases}

First, we calculate the internal energy or the enthalpy of glassy phases as a simple index indicating the stability of glassy phases. Figure 4 shows the concentration dependence of the atomic enthalpy of the glassy phases obtained by quenching at a cooling rate $2 \times 10^{-4}$, where the closed triangles and the open triangles correspond to the system of the atomic size ratio 0.8 and 0.85 , respectively. Figure 4 shows the following three features: (1) The energy of the glassy state is lower for the system with larger atomic size difference at compositions except for $x_{2}=0.2$. (2) The minimum locus of the enthalpy can be seen at the compositions deviating from $x_{2}=0.5$ to a higher composition. (3) The order of the deviation increases with increasing atomic size mismatch, which is exemplified by the fact that the glassy state has the lowest energy at $x_{2}=0.7$ for $r_{2}=0.8$.

Supposing that the lower enthalpy of glassy phase means the higher stability of that phase, we come to our first conjecture: The glass-forming ability is higher when the enthalpy of the glassy state is lower. Permitting this conjecture, we can estimate the glass-forming ability of the systems inside the region that is previously shown as region (C) in Fig. 1, as shown in Fig. 5. In Fig. 5, it is to be noted that the most interesting feature is that the asymmetry for the composition, which is also shown in Fig. 1 slightly, tends to be enhanced in the systems with larger atomic size difference. We expect that the scheme solely based on the enthalpy of the glassy phases would be one of possible candidates for criterion for estimating the glass-forming ability by MD simulations.

\subsubsection{Diffusivity of liquid phases}

Followed by the previous estimation based on the enthalpy with a purely kinematical nature, we next proceed to the estimation from a dynamical aspect by focusing on the atomic diffusion in liquid phases. On the basis of the 


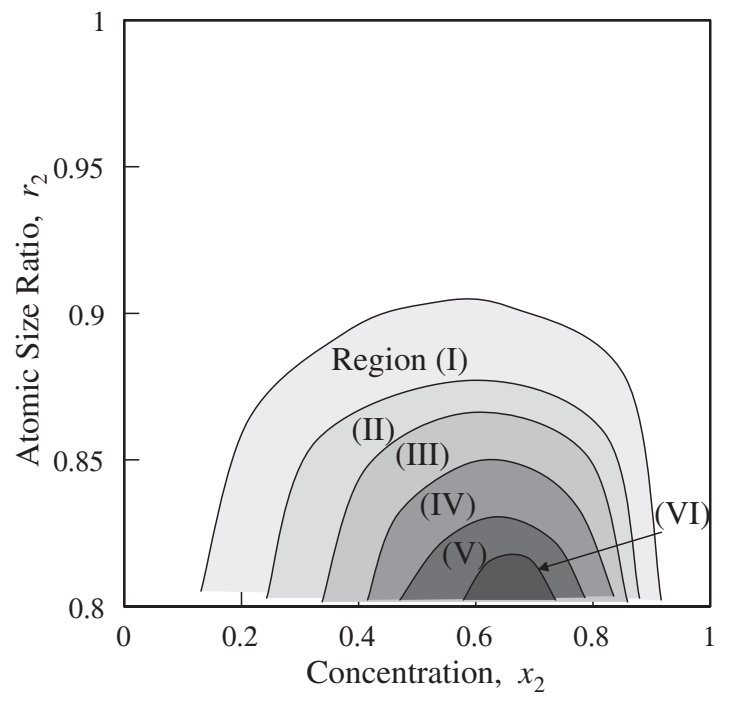

Fig. 5 The glass-forming ability of the model binary system estimated from the enthalpy of the glassy phases obtained at a cooling rate $\left(2 \times 10^{-4}\right)$. In the regions (I)-(VI), the glass-forming ability is expected to become higher from (I) to (VI) in ascending order.

expectation that the atomic diffusion should be suppressed in a dense liquid, we take the following procedures. We first estimate the density of liquid phases by considering the atoms as hard spheres with a diameter proportional to their LJ radius, and then calculate the atomic diffusivity in the liquid phases, and then compare the density with the atomic diffusivity.

In simulations, the diameter of atom is defined as the nearest neighbor distance of the stable crystalline phase (fcc) for the single element system at $T=0$. Then we define the packing density as the volume fraction of atoms by regarding them as hard spheres. We also calculate the diffusion constants by the time evolution of the atomic mean square displacements. The calculation results for the supercooled liquid phases at $T=0.4$ for the $r_{2}=0.8$ system are shown in Fig. 6. As expected, the diffusion rate is drastically suppressed more than $30 \%$ by the slight enhancement of packing density less than $1 \%$. We expect that the rate of the liquid-tocrystal or the glass-to-crystal transition should be also suppressed in high packing phases so the glass-forming ability would be enhanced in such phases. Thus we come to our second conjecture: The glass-forming ability is higher when the packing density of the liquid phase is higher.

Figure 7 shows the estimation of the glass-forming ability on the basis of the packing density of liquid phases at $T=0.70$. This result is considerably similar to the previous one and the asymmetry of glass-forming region with respect to $x_{2}$ is enhanced for large atomic size-mismatch systems as found in Fig. 5.

\subsubsection{Relaxation behavior of liquid and glassy phases}

In the next stage, we try to estimate the stability of glassy and supercooled liquid phases by evaluating the relaxation behavior from a viewpoint based on the energy landscape theory. According to the idea of the energy landscape, we here propose the following interpretation for the rapid solidification process, which is schematically illustrated in Fig. 8. By quenching from equilibrium liquid phases at a

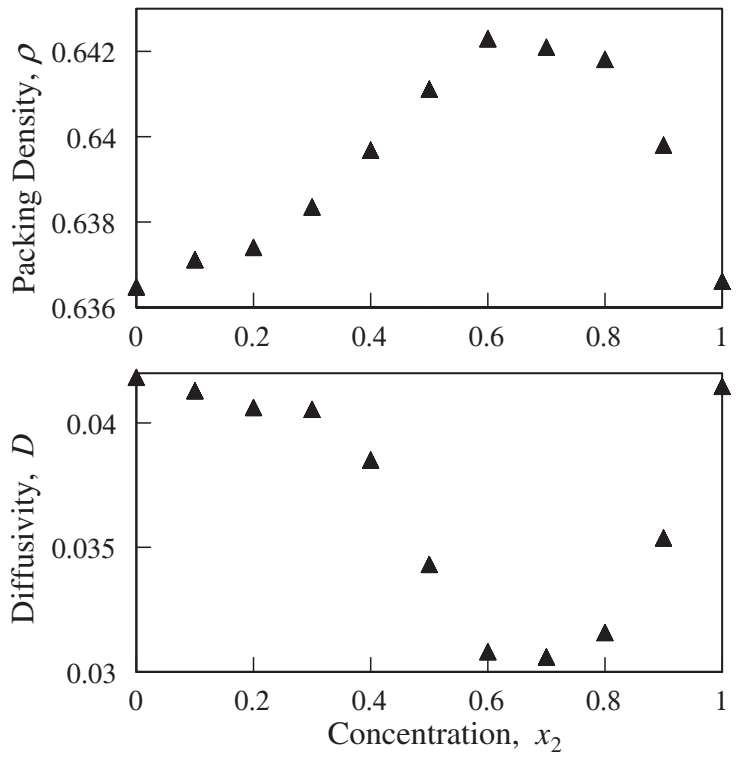

Fig. 6 The concentration dependence of the packing density (upper column) and that of the atomic diffusivity (bottom column) of the supercooled liquid phases at $T=0.4$ for the system with the atomic size ratio 0.8 .

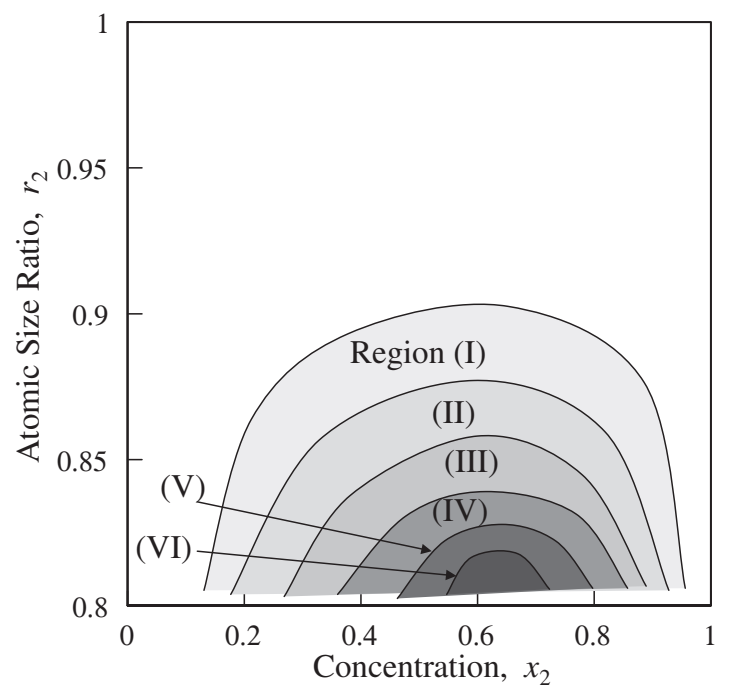

Fig. 7 The glass-forming ability of the model binary system estimated from the packing density of the liquid phases at $T=0.7$. In the regions (I)(VI), the glass-forming ability is expected to become higher from (I) to (VI) in ascending order.

sufficiently high cooling rate, the system can be easily trapped into one of basins with high enthalpy $E$, because the number $\Omega(E)$ of such basins is much larger than that of basins with lower enthalpy. On the other hand, when the cooling rate is considerably low but not so low as crystallization occurs, the system would fall into a basin with lower enthalpy near the ideal glassy state via a sequence of metastable supercooled liquid phases. In a similar sense, the relaxation processes of both supercooled liquid and glassy phases are understood as transitions from high enthalpy and high entropy phases to low enthalpy and low entropy phases, as shown in Fig. 8. To confirm this interpretation based on the energy landscape theory, we shall investigate the relaxation 


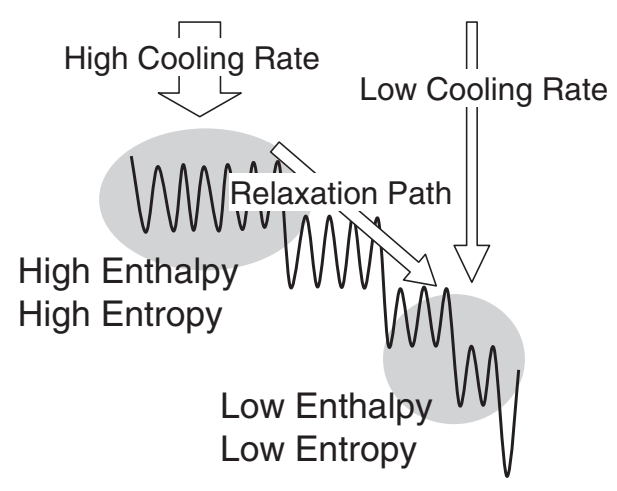

Fig. 8 The schematic view of rapid solidification processes and relaxation processes in the framework of the energy landscape theory.

behavior at the atomistic scale by calculating two parameters that indicate the local atomic structure.

The first is a parameter $N_{\text {free }}$, defined by the number of atoms with the coordination number less than twelve, which reflects the amount of the free volume of the system. The atom with less neighbors should have an excess free volume, and $N_{\text {free }}$ is shown by the earlier simulation study ${ }^{10)}$ to be a good parameter that indicates the atomic free volume. A large excess free volume indicates high possibility of atom configuration or, equivalently, high configurational entropy. So the parameter $N_{\text {free }}$ is expected to serve as an index of the configurational entropy.

The second is $N_{\text {icosa }}$, defined by the number of icosahedral clusters, which indicates the local dense packing structure, and can be used as an index of local density and 'ideality' of the glassy phase. The $N_{\text {icosa }}$ is introduced in the present analysis because of the consideration that the basic unit of the glassy phases in the binary systems with not-so-large size differences is the icosahedral cluster. ${ }^{11)}$ Thus, we expect that the number of icosahedral clusters in the system should increase when the state approaches the ideal glassy state. Under this expectation, we investigate the local symmetry by calculating the Voronoi polyhedron ${ }^{12)}$ of each atom, and evaluate the number $N_{\text {icosa }}$ of atoms that are centered at the icosahedral clusters.

By calculating the above two parameters, we can investigate the relaxation behavior of supercooled liquids and glassy phases at the atomistic scale. Figure 9 shows the isothermal calculation results for a supercooled liquid phase of the $\left(r_{2}, x_{2}\right)=(0.9,0.5)$ system quenched from $T=0.35$ and then annealed at $T=0.30$. The atomic enthalpy and the free volume decrease at the first stage, and then seem to fluctuate in the succeeding stages. The number of icosahedral clusters rises at the first stage and then also fluctuates. The former stages clearly correspond to a relaxation period, in which the enthalpy and the entropy of the system decrease from a higher state to a lower state. On the other hand, the latter periods seem to correspond to the quasi-equilibrium state dynamically going around a part of the configuration space within one of megabasins.

In order to investigate the relaxation behavior of glassy phases at longer time stages, we shall examine the structural difference between glassy phases at different cooling rates by calculating the parameters introduced above. This is because

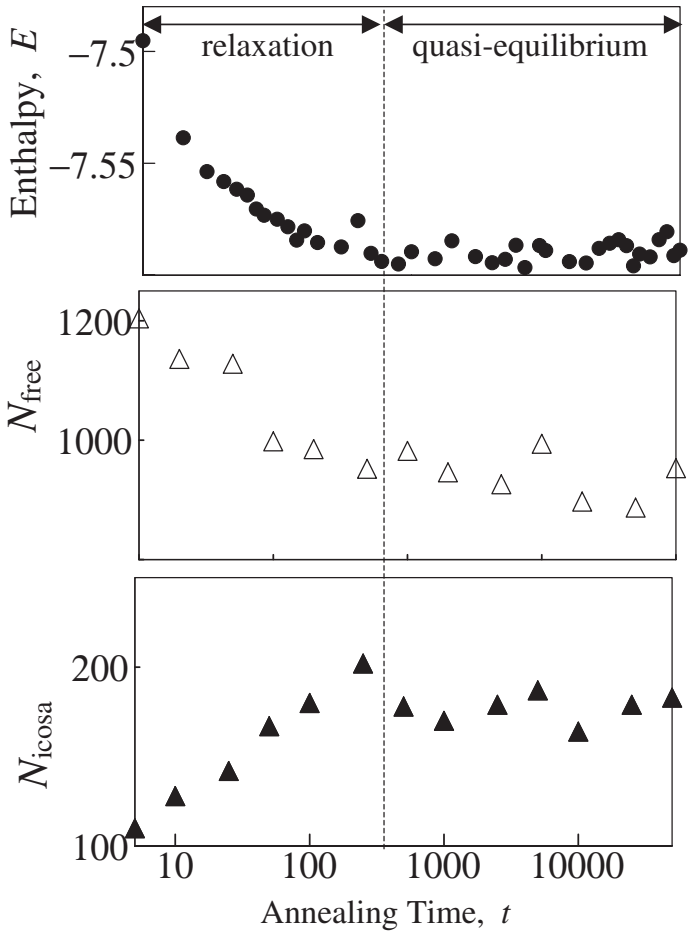

Fig. 9 The time evolution of the atomic enthalpy (closed circles), the number $N_{\text {free }}$ of atoms with large free volume (open triangles), and the number $N_{\text {icosa }}$ of icosahedral clusters (closed triangles) of the supercooled liquid phase of the $\left(r_{2}, x_{2}\right)=(0.9,0.5)$ system under the annealing at $T=0.3$.

we consider that the lower cooling rate can be equivalent to the longer relaxation period at an isothermal annealing. Figure 10 shows the enthalpy, the atomic free volume, and the number of icosahedral clusters calculated for the glassy phases obtained by different cooling rates ranging from $5 \times$ $10^{-6}$ to $4 \times 10^{-3}$ for the $\left(r_{2}, x_{2}\right)=(0.8,0.5)$ system. As the cooling time increases, or as the cooling rate decreases, the corresponding glassy phase tends to have a lower enthalpy, a smaller free volume, and more icosahedral clusters. In other words, the relaxation proceeds from glassy phases with lower packing and higher enthalpy to those with higher packing and lower enthalpy.

As seen from the dependence of the parameters on the cooling rate in Fig. 10, even at the lowest cooling rate, the glassy phase has not yet reached the fully relaxed state. This means that the system is still far from the ideal glassy state. The timescale necessary to reach the ideal glass is determined by kinematical factors just like the shape of energy landscape of the system, as well as dynamical factors like atomic diffusivity. All these factors should be reflected in the relaxation behavior shown in Fig. 10. Therefore, if we would estimate the relaxation timescale of the system by the relaxation behavior, we could evaluate the glass-forming ability of the system by relating with the relaxation timescale. In other words, in the system with large glass-forming ability, the shape of megabasin in the energy landscape corresponding to glassy phases should be deep and wide and/or the atomic diffusivity should be considerably low in supercooled liquid and glassy phases, both of which make the relaxation time toward the ideal glass be long. Thus, in next section, we 

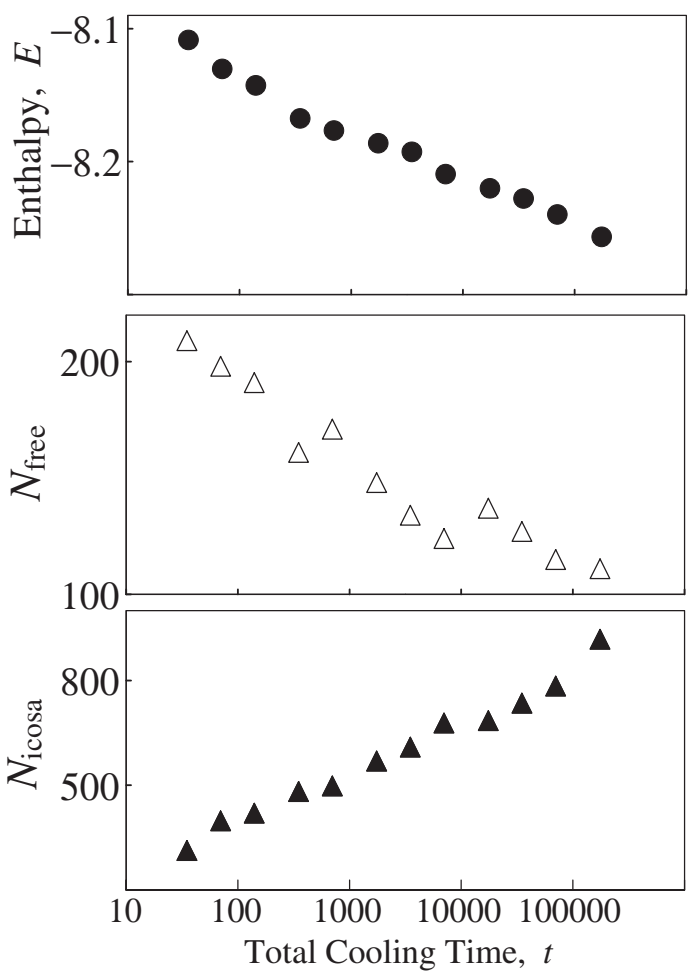

Fig. 10 The cooling rate dependences of the atomic enthalpy (closed circles), $N_{\text {free }}$ (open triangles), and $N_{\text {icosa }}$ (closed triangles) of the asquenched glassy phase of the $\left(r_{2}, x_{2}\right)=(0.8,0.5)$ system.

shall estimate the relaxation time as a probe for the glassforming ability by calculating the dependence of the glass transition temperature on the cooling rate.

\subsection{Cooling rate dependence of $\boldsymbol{T}_{\mathrm{g}}$}

Figure 11 shows the temperature dependences of the atomic volume in the rapid cooling procedures at two different cooling rates $\left(2 \times 10^{-5}\right.$ and $\left.2 \times 10^{-3}\right)$ for the $\left(r_{2}\right.$, $\left.x_{2}\right)=(0.8,0.5)$ system. We here define the glass transition temperature $T_{\mathrm{g}}$ by the point at discontinuity in the volume change as depicted in Fig. 11. Figure 11 shows the fact that the estimated value of $T_{\mathrm{g}}$ depends on the cooling rate.

Under the above definition of the glass transition temperature $T_{\mathrm{g}}$, we can calculate the cooling rate dependence of $T_{\mathrm{g}}$. The results calculated for the $\left(r_{2}, x_{2}\right)=(0.9,0.7)$ system are shown in Fig. 12(a). Note that we can observe crystallization in this system at the lowest cooling rate $5 \times 10^{-6}$. The glass transition temperature decreases with decreasing the cooling rate $R$. In addition, around the critical cooling rate $\left(1 \times 10^{-5}\right)$ for glass-formation, the dependence of $T_{\mathrm{g}}$ becomes almost saturated. This implies that the system would approach to the ideal glass or the bottom of a crater in the energy landscape.

We have also shown the cooling rate dependence of $T_{\mathrm{g}}$ calculated for the two systems with the atomic size ratio $r_{2}=0.8$ in Fig. 12(b). Here the open symbols and the closed symbols correspond to the $\left(r_{2}, x_{2}\right)=(0.8,0.5)$ system and the $(0.8,0.2)$ system, respectively. In each case, we have never observed crystallization in the simulation time. For the system of $x_{2}=0.5, T_{\mathrm{g}}$ continuously tends to decrease with decreasing $R$. On the other hand, $T_{\mathrm{g}}$ tends to be saturated for the system of $x_{2}=0.2$. The difference in tendency suggests

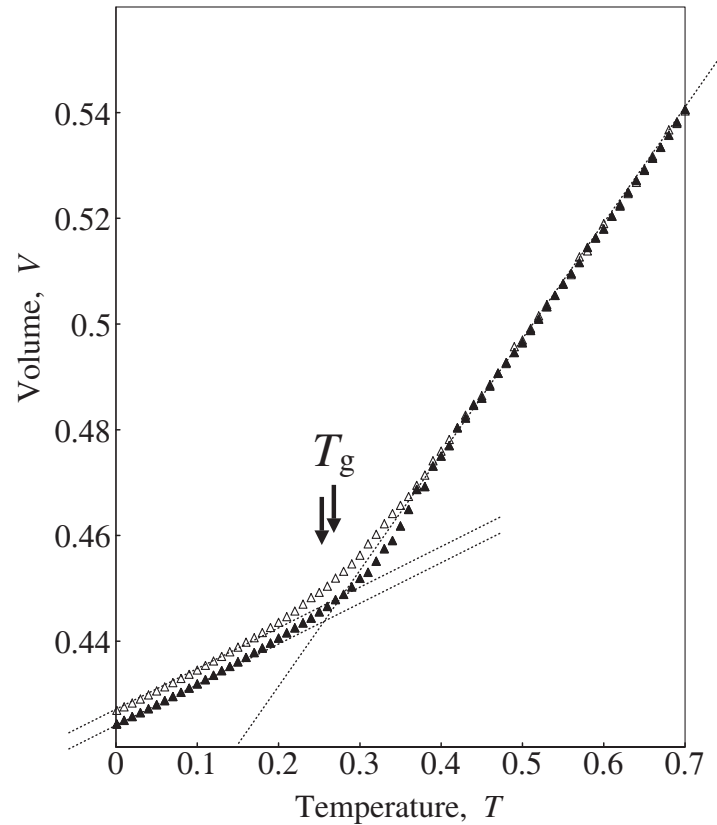

Fig. 11 The time evolution of the atomic volume of the $\left(r_{2}, x_{2}\right)=(0.8,0.5)$ system under rapid solidification at different cooling rates, where the open triangles and the closed triangles correspond to the higher cooling rate $\left(2 \times 10^{-3}\right)$ and the lower rate $\left(2 \times 10^{-5}\right)$, respectively.
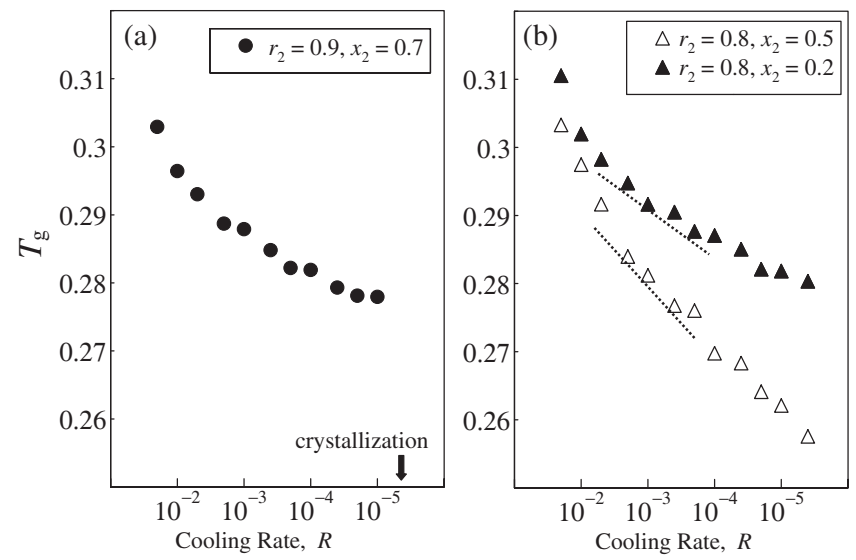

Fig. 12 The cooling rate dependences of the glass transition temperature $T_{\mathrm{g}}$ for the $\left(r_{2}, x_{2}\right)=(0.9,0.7)$ system (a) and the two $r_{2}=0.8$ systems (b), where the open triangles and the closed triangles correspond to the $x_{2}=0.5$ system and the $x_{2}=0.2$ system, respectively. Note that the cooling rate is expressed in descending order.

that the timescale for relaxation should be shorter and the glass-forming ability might be smaller in the latter system. Thus we come to our third conjecture: If the glass transition temperature has a large dependence on the cooling rate, the glassy state is far from the ideal glass, and accordingly the glass-forming ability of the system is estimated to be high.

Unfortunately, we have not reached the point where we could propose a specific analytical form describing the cooling rate dependence of $T_{\mathrm{g}}$. Therefore, we calculate the dependence $\Delta T_{\mathrm{g}} / \Delta R$ at a common cooling rate $R=1 \times$ $10^{-3}$, which corresponds to the slope of the dotted lines depicted in Fig. 12(b), by taking the slope as a measure for the glass-forming ability. Thus we have a map of the glassforming ability predicted by the cooling rate dependence of 


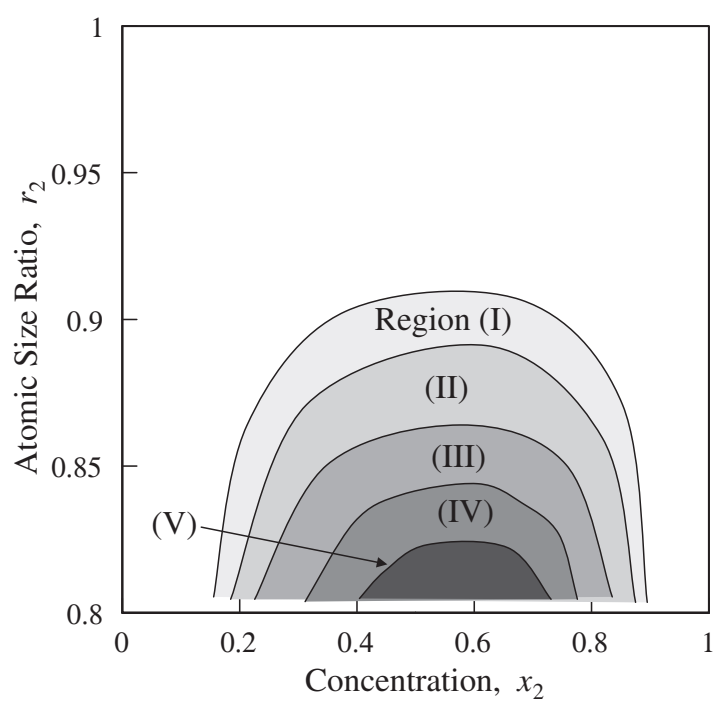

Fig. 13 The glass-forming ability of the model binary system estimated from the cooling rate dependence of the glass transition temperature. In the regions (I)-(V), the glass-forming ability is expected to become higher from (I) to (V) in ascending order.

$T_{\mathrm{g}}$ as shown in Fig. 13. We can find the asymmetry on the composition again, but the order of asymmetry is not so large as found in the earlier two estimations shown in Figs. 5 and 7.

\section{Discussion}

In the present study, we have proposed three criteria to estimate the glass-forming ability: the enthalpy of glassy phases, the packing density of liquid phases, and the cooling rate dependence of the glass transition temperature. In this section, the above criteria are criticized by the following factors: stability of solid solution with respect to phase equilibria, and internal stress. The reason why we shall discuss the stability of solid solution is as follows. Because the simulation time is not so long as ordered crystalline phases would form resulting from long-range atomic diffusion, the firstly appearing phase at the primary crystallization stage is expected to be a solid solution. Therefore, relative stability between the solid solution phases and the glassy or the liquid phases for the concerning system should be an important factor in determining the glass-forming ability of the system. Indeed, Egami and Waseda, ${ }^{13)}$ and Takeuchi and Inoue $^{14)}$ have shown that the amorphous-forming ranges experimentally observed for alloy systems can be well understood by analyzing the stability of solid solution phases against glassy phases.

In the simple systems we have treated here, it is shown ${ }^{4)}$ that the fcc solid solution is the most stable phase among solid solutions for almost parameter sets in the $\left(r_{2}, x_{2}\right)$ space for $r_{2}>0.8$. Therefore, we focus on the stability of fcc solid solutions. In rapid solidification processes from liquid phase, crystallization always takes place in a supercooled liquid phase above $T_{\mathrm{g}}$. Accordingly, the phase stability of solid solutions against supercooled liquid phases should play a crucial role in the crystallization stage. The melting temperature directly indicates the point where the solid phase becomes unstable against the liquid phase. Thus, we shall

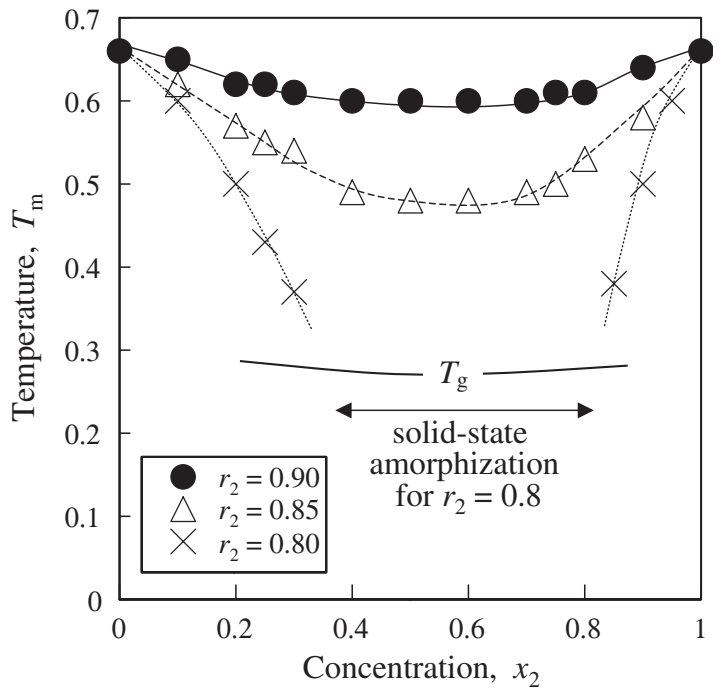

Fig. 14 The concentration dependences of the melting temperature $T_{\mathrm{m}}$ for fcc solid solutions, where the circles, triangles, and crosses correspond to the systems with the atomic size ratio $r_{2}=0.9,0.85$, and 0.8 , respectively. The concentration dependence of $T_{\mathrm{g}}$ for the $r_{2}=0.8$ system at a cooling rate $2 \times 10^{-4}$ is depicted by the bold line. For the system with $r_{2}=0.8$, solid solutions directly transform into glassy phases by solid-state amorphization below $T_{\mathrm{g}}$ in the concentration range from $x_{2}=0.35$ to 0.8 .

calculate the concentration dependence of the melting temperature of fcc solid solutions by the MD simulation as a measure of the phase stability of solid solutions.

The calculation procedure is as follows: We first prepare fcc solid solutions with the concentration ranging from $x_{2}=$ 0 to 1 for the systems with the atomic size ratio $r_{2}=0.9$, 0.85 , and 0.8 at the temperature $T=0$. Then we gradually heat the system at a heating rate $2 \times 10^{-5}$ to melt. By monitoring the atomic volume, the enthalpy, and the diffusivity of the system, we can recognize a crystal-toliquid transition by a sudden jump in these properties. ${ }^{10)}$ Thus, we can estimate the melting temperature $T_{\mathrm{m}}$ of the fcc solid solution in the system.

The calculated results are shown as Fig. 14. Here the circles, the triangles, and crosses indicate the melting temperatures of the solid solutions with the atomic size ratio $r_{2}=0.9,0.85$, and 0.8 , respectively, and the liquidus lines are drawn as a visual guide. For the system with the atomic size ratio $r_{2}=0.8$, the solid solutions are not stable and directly transform into glassy phases even at $T=0$ in the composition range from $x_{2}=0.35$ to 0.80 . This is the reason why we could not calculate the melting temperature of fcc solid solution in this composition range. In Fig. 14, we have also depicted a concentration dependence of $T_{\mathrm{g}}$ calculated in the case of solidification at a cooling rate $2 \times 10^{-4}$ for the $r_{2}=0.8$ system by a bold line. The above-mentioned transformation from a solid solution into a glassy phase in the $r_{2}=0.8$ system takes place far below $T_{\mathrm{g}}$ and can be regarded as solid-state amorphization. This implies that the glassy phases should be more stable than the solid solution phases in the system with a large atomic size difference such as $r_{2}=0.8$. By a similar MD calculation ${ }^{4)}$ on the same model studied here, it is shown that there is a wide composition range where glassy phases actually have lower energy than 
solid solutions in the system with a large atomic size difference as $r_{2} \leq 0.8$. This fact suggests that, in the systems with large atomic size differences, we should take ordered phases such as metallic compounds into account in order to estimate the phase stability of glassy or liquid phases against crystallization correctly. However, this point is out of the scope of the present study and one of future problems.

In addition to the instability of solid solution phases for the $r_{2}=0.8$ system in the composition range $x_{2}=0.35-0.8$, Fig. 14 shows a tendency that the minimum locus of liquidus lines shifts from $x_{2}=0.5$ to a higher composition as the atomic size difference goes larger. The solid solution is expected to be most unstable at the composition corresponding to the minimum locus of $T_{\mathrm{m}}$. In this context, the calculation results seem to support the enhanced asymmetry with respect to $x_{2}$ of the predicted glass-forming ability for the large atomic size mismatched systems found in Figs. 5, 7, and 13 . We note that, experimentally, in many cases the maximum glass-forming ability is attained near the eutectic composition, which corresponds to the locus of the lowest liquidus temperature.

We next discuss the stability of solid solutions from a viewpoint of the atomic level stress. Egami and Waseda have proposed $^{13)}$ a criterion for the amorphous-formation of alloys based on the atomic level stress of solid solutions and elegantly explained the glass-forming range for both metalmetal and metal-metalloid binary systems by using their theory. Following their schemes, the local atomic level stress $\lambda$ of solid solutions caused by alloying a different size element can be calculated as a function of the atomic size ratio. Egami and Waseda suggested that solid solutions would become unstable and undergo amorphization when the local atomic level stress of the system reaches some critical value $\lambda_{\mathrm{c}}$. The theoretical value of $\lambda_{\mathrm{c}}$ for fcc solid solutions is 0.0625 and an empirical value of $\lambda_{c}$ fitting from the experimental data is $0.0539 .{ }^{13)}$ Figure 15 shows the calculated dependence of the stress $\lambda$ on the composition and the

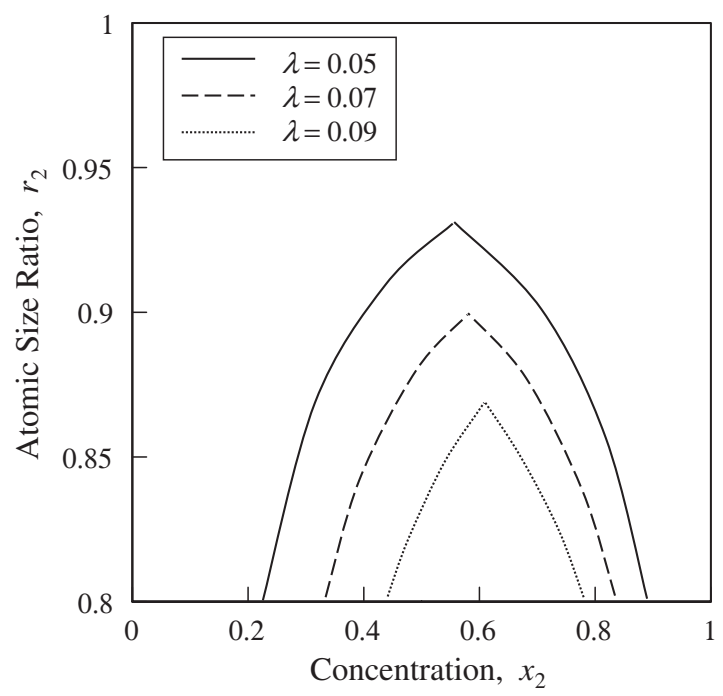

Fig. 15 The trajectories of the constant atomic stress $\lambda$ of solid solutions depicted in the $\left(x_{2}, r_{2}\right)$ plane. The solid line, the dashed line, and the dotted line correspond to $\lambda=0.05,0.07$, and 0.09 , respectively. atomic size ratio of alloying elements. The solid line, the dashed line, and the dotted line correspond to the trajectory for $\lambda=0.05,0.07$, and 0.09 , respectively. In consideration of the fact that the higher value of the local strain implies the lower stability of the solid solution, Fig. 15 indicates that the lowest stability region shifts from $x_{2}=0.5$ to a higher compositions with increasing the atomic size difference. Therefore, the order of instability of the solid solution phases predicted by their theory shows completely the same trends as the glass-forming ability predicted by our calculations. We note that, for the systems with the atomic size ratio of 0.8 , the instability region from $x_{2}=0.35$ to 0.8 of the fcc solid solution found in Fig. 14 almost coincides with the high stress region in Fig. 15, which demonstrates the validity of their theory.

Here we should also mention the local atomic level stresses in the glassy phases. Egami and Waseda theory is based on solely the atomic level stress in the solid solution phases and implicitly neglect the atomic level stress in glassy phases. Even in the more sophisticated version of this theory proposed by Takeuchi and Inoue, ${ }^{14)}$ the elastic enthalpy of glassy state due to internal stress is neglected, although five kinds of enthalpy terms are taken into account. However, Egami and Waseda theory reproduces the glass-forming range for more than 50 binary alloy systems and Takeuchi and Inoue theory reproduces the glass-forming range for numerous ternary alloy systems. This fact implies that the assumption that the local atomic level stress in glassy phases can be neglected should be a good approximation. This assumption is also consistent with the results of an MD study, ${ }^{15)}$ which has reported that the atomic stresses considerably get relaxed in glassy state in comparison with crystalline state after solid-state amorphization for a binary alloy system with a large atomic size ratio 1.45.

Although our prediction of the glass-forming ability of the model systems cannot verified by the direct simulations due to the computational capacity, the relative phase stability between the glassy phases or the liquid phases and the solid solution phases agrees to the conclusion. Thus, the above discussion offers a sort of circumstantial evidence.

\section{Summary}

Three criteria for estimating the glass-forming ability accessible by the MD simulations are proposed. The first is the enthalpy of glassy phases (a purely kinematical factor), the second is the packing density of liquid phases, which is based on the diffusivity of liquid phases (a purely dynamical factor), and the third is the cooling rate dependence of the glass transition temperature, which is based on the relaxation behavior of liquid and glassy phases (both kinematical and dynamical factor). All these three approximately give the same prediction for the glass-forming ability with respect to the point that the asymmetry for the composition of the glassforming range would be enhanced in the systems with large atomic size differences. The prediction for the glass-forming ability by the MD simulation is consistent with stability analyses of solid solution phases against liquid phases or glassy phases with respect to phase equilibria and internal stress. Atomic level analyses show that the structural 
relaxation always proceeds accompanied with the increase of the icosahedral structure in both glassy phases and supercooled liquid phases. This relaxation behavior can be naturally understood in the framework of the energy landscape theory.

The molecular dynamics simulation is a very powerful tool to investigate dynamical processes at the atomistic scale, but the restriction due to the time scale is very severe in making predictions for phenomena with macroscopic time scales. However, we can pick up some essence applicable to long timescale from some characteristics that are obtained in a calculation time. It looks like a sort of an 'acceleration experiment' with respect to time and we hope that the similar ideas as proposed here would be fruitful for the field of computational materials science.

\section{Acknowledgements}

The present research is partly supported by Grant-in-Aid for Scientific Research (No. 428-15074220) from Japan Society for the Promotion of Science. The numerical calculations were performed on NEC SX-5 (Numerical
Materials Simulator) at National Institute for Materials Science.

\section{REFERENCES}

1) A. Inoue, T. Zhang and T. Masumoto: Mater. Trans., JIM 31 (1990) $177-183$.

2) A. Peker and W. L. Johnson: Appl. Phys. Lett. 63 (1993) 2342-2344.

3) A. Inoue: Bulk Amorphous Alloys: Preparation and Fundamental Characteristics, (Trans Tech Pub., Zurich, 1998) pp2-10.

4) M. Shimono and H. Onodera: Scr. Mater. 44 (2001) 1595-1598.

5) F. H. Stillinger: Science 267 (1995) 1935-1939.

6) P. G. Debenedetti and F. H. Stillinger: Nature 410 (2001) 259-267.

7) H. C. Andersen: J. Chem. Phys. 72 (1980) 2384-2393.

8) J. M. Sanchez, J. R. Barefoot, R. N. Jarrett and J. K. Tien: Acta Metall. 32 (1984) 1519-1525.

9) S. Sastry, P. G. Debenedetti and F. H. Stillinger: Nature 393 (1998) 554-557.

10) M. Shimono and H. Onodera: Mater. Sci. Eng. A 304-306 (2001) 515519.

11) M. Shimono and H. Onodera: Mater. Trans., JIM 39 (1998) 147-153.

12) J. L. Finney: Proc. R. Soc. London Ser. A 319 (1970) 479-493.

13) T. Egami and Y. Waseda: J. Non-Cryst. Solids 64 (1984) 113-134.

14) A. Takeuchi and A. Inoue: Mater. Trans. 42 (2001) 1435-1444.

15) M. Katagiri and H. Onodera: Mater. Trans., JIM 40 (1999) 1274-1280. 